\title{
Remote control of restricted sets of operations: Teleportation of Angles
}

\author{
S.F. Huelga ${ }^{1}$, M.B. Plenio ${ }^{2}$ and J.A. Vaccaro ${ }^{1}$ \\ ${ }^{1}$ Department of Physical Sciences, University of Hertfordshire, Hatfield AL10 9AB, UK \\ ${ }^{2}$ QOLS, Blackett Laboratory, Imperial College of Science, Technology and Medicine, London, SWr $2 B W, U K$
}

(February 1, 2008)

\begin{abstract}
We study the remote implementation of a unitary transformation on a qubit. We show the existence of non-trivial protocols (i.e., using less resources than bidirectional state teleportation) which allow the perfect remote implementation of certain continuous sets of quantum operations. We prove that, up to a local change of basis, only two subsets exist that can be implemented remotely with a non-trivial protocol: Arbitrary rotations around a fixed direction $\vec{n}$ and rotations by a fixed angle around an arbitrary direction lying in a plane orthogonal to $\vec{n}$. The overall classical information and distributed entanglement cost required for the remote implementation depends on whether it is a priori known to which of the two teleportable subsets the transformation belongs to. If it is so, the optimal protocol consumes one e-bit of entanglement and one c-bit in each direction. If the subset is not known, two e-bits of entanglement need to be consumed while the classical channel becomes asymmetric, two c-bits are conveyed from Alice to Bob but only one from Bob to Alice.
\end{abstract}

PACS-numbers: 03.67.-a, 03.65.Bz

\section{INTRODUCTION}

Using entanglement as a resource is a common feature of many tasks in quantum information processing [1]. A canonical example of entanglement-assisted processes is provided by quantum state teleportation [2], where an arbitrary qubit state can be transferred with perfect fidelity among distant parties with the sole use of two classical bits (c-bits) and the consumption of a distributed maximally entangled state, i.e., one e-bit of shared entanglement. Recently we have a addressed a related problem where the aim is to teleport across distant parties not a quantum state but a quantum operation [3]. By this we mean the following. Alice and Bob are set in remote locations and one of the parties, say Alice, is given a black box with the ability of performing a very large set of unitary transformations $U$ on a qubit. The requirement of the set of allowed transformations being very large is imposed with the aim of excluding, by construction, the possibility of teleporting the full black box to Bob, which would exhaust entanglement resources very quickly. We will say that the operation $U$ has been teleported to Bob, or equivalently, it has been remotely implemented if, for any qubit state Bob may hold, a protocol involving only local quantum operations and exchange of classical communication (LQCC) can yield a final global state where Bob holds the state transformed by the operation $U$ disentangled from any other system (See below for a quantitative formulation). Our previous results show that if we want the transformation $U$ to be an arbitrary element of the group $S U(2)$, no LQCC protocol can exist consuming less overall resources than teleporting Bob's state to Alice followed by Alice teleporting the state transformed by $U$ back to Bob. In other words, the remote implementation of an arbitrary unitary operation on a qubit cannot be accomplished by means of any local protocol which uses less resources than bidirectional quantum state teleportation (BQST). This amounts to two e-bits of entanglement and two classical bit in each direction. The ultimate responsible for this result is linearity. Therefore, the impossibility of implementing remotely an arbitrary $U$ without resorting to state transfer belongs to the family of no-go results imposed by the linear structure of quantum mechanics and exemplified, for instance, by the non-cloning theorem [4.

What happens if the requirement of being able to implement any $U$ is relaxed? Can we find families of operators that can be implemented consuming less overall resources than BQST?. We should stress that we are interested here in exploiting entanglement, therefore any strategy which attempts the local reconstruction of $U$ [5] is excluded from our valid protocols. In addition, we want to keep to a minimum the available a priori information about $U$. Note, in particular, that if both the form of $U$ and Bob's initial state are completely known, the posed problem reduces to remote state preparation [6]. Finally, we want the procedure to work with perfect efficiency. Imperfect storage of quantum operations have been recently discussed by Vidal et al. [7]. We will show that there are indeed two restricted classes of operations that can be implemented remotely using less overall resources than BQST and only two (up to a local change of basis). These are arbitrary rotations around a fixed direction $\vec{n}$ and rotations by a fixed angle around an arbitrary direction lying in a plane orthogonal to $\vec{n}$. 
We have organized the paper in seven further sections. Section II revises the necessary resources for achieving the remote implementation of an arbitrary $U$. In section III a LQCC protocol exhausting these resources and achieving the maximum probability of success allowed for arbitrary $U$ is constructed. Remarkably, two possible sets of transformations could be implemented accurately with this procedure, as discussed in section IV. A geometrical picture of why it is possible to engineer a final correction step in these cases is presented in section V. The uniqueness of the subsets is proven in section VI, the technical bulk of this paper. Section VII deals with the resources trade-off when some a priori information about the functional form of the transformation $U$ is provided. Final section VIII ends summarizes the results and end up with proposing an experimental scenario where the teleportation of angles could be demonstrated.

\section{REMOTE IMPLEMENTATION OF AN ARBITRARY $U$ : NECESSARY RESOURCES}

Assuming the black box to be a classical system, we are seeking a protocol with the following structure [3]

$$
G_{2} U G_{1}\left(|\chi\rangle_{a A B} \otimes|\psi\rangle_{b}\right)=|\Phi(\chi)\rangle_{a A B} \otimes U|\psi\rangle_{b}
$$

where certain fixed operations $G_{1}$ and $G_{2}$ are performed, respectively, prior to and following the action of the arbitrary $U$ on a qubit $a$ on Alice's side. The fact that the operation $G_{1}$ has to be non-trivial follows from the results of Nielsen and Chuang when analyzing universal programmable gates [8]. We assume that Alice and Bob share initially some entanglement, represented by the joint state $|\chi\rangle_{\alpha A B}$. The purpose of the protocol is to end up with Bob holding a qubit in the transformed state $U|\psi\rangle_{b}$, for any initial state $|\psi\rangle_{b}$ and with perfect efficiency. Note that the final distributed state involving the remaining subsystems $a A B$ is independent of both $U$ and $|\psi\rangle_{b}$ [3]. As in [3], it will convenient to use a nonlocal unitary representation of the transformation, with $G_{1}$ and $G_{2}$ being unitary operators acting on possibly all subsystems. For instance, a possible solution, while in principle not necessarily optimal, corresponds to each $G_{i}$ being a state teleportation process. In the following we will establish lower bounds on the amount of classical communication and the amount of entanglement required for the teleportation of an arbitrary unitary transformation. Our argument employs the principle that entanglement cannot be increased under LQCC to show that 2 e-bits are necessary and it uses the impossibility of superluminal communication to demonstrate that 2 classical bits have to be sent from Alice to Bob and at least one bit has to be transferred from Bob to Alice.

Assume that we could teleport any arbitrary operation $U$ from Alice to Bob. Therefore, a universal protocol involving operations $G_{1}$ and $G_{2}$ would yield the outcome $|\Phi(\chi)\rangle_{a A B} \otimes U|\psi\rangle_{b}$, independently of the actual form of $U$. It is easy to show that then it would also be possible to implement remotely an arbitrary controlled-U gate. By this we mean that the remote implementation of $U$ is performed conditional on the state of certain control qubit $c$, so that the action of the black box is to apply the identity if the control qubit is state $|0\rangle_{c}$ and to apply $U$ when the control bit is state $|1\rangle_{c}$. That is, Eq.(11) is replaced by

$$
G_{2} U_{c} G_{1}\left(|\chi\rangle_{a A B} \otimes|\psi\rangle_{b}\right)=|\Phi(\chi)\rangle_{a A B} \otimes\left(c_{0}|0\rangle_{c} \otimes \mathbb{1}|\psi\rangle_{b}+c_{1}|1\rangle_{c} \otimes U|\psi\rangle_{b}\right),
$$

where

$$
U_{C}=|0\rangle_{c c}\langle 0|\otimes \mathbb{1}+| 1\rangle_{c c}\langle 1| \otimes U
$$

and $|c\rangle=c_{0}|0\rangle_{c}+c_{1}|1\rangle_{c}$ is an arbitrary state of the control qubit, which, without loss of generality, can be assumed to be part of the black box and therefore unaffected by the action of the operations $G_{i},(i=1,2)$. Let us decompose the global state after the application of $G_{1}$ as follows

$$
|c\rangle \otimes G_{1}\left(|\chi\rangle_{a A B} \otimes|\psi\rangle_{b}\right)=\left(c_{0}|0\rangle_{c}+c_{1}|1\rangle_{c}\right) \otimes\left(|0\rangle_{a}|\xi\rangle_{0}+|1\rangle_{a}|\xi\rangle_{1}\right)
$$

where the, possibly distributed, states $|\psi\rangle_{i}$ are neither necessarily orthogonal not normalized. The action of $U_{c}$ brings this state onto

$$
U_{c}\left(c_{0}|0\rangle_{c}+b|1\rangle_{c}\right) \otimes\left(|0\rangle_{a}|\xi\rangle_{0}+|1\rangle_{a}|\xi\rangle_{1}\right)=c_{0}|0\rangle_{c}\left(\mathbb{1}|0\rangle_{a}|\xi\rangle_{0}+\mathbb{1}|1\rangle_{a}|\xi\rangle_{1}\right)+c_{1}|1\rangle_{c}\left(U|0\rangle_{a}|\xi\rangle_{0}+U|1\rangle_{a}|\xi\rangle_{1}\right)
$$

Now, the subsequent action of the operation $G_{2}$ gives the transformation law Eq.(2) provided that Eq.(11) holds for every qubit transformation $U$.

A simple controlled- $U$ operation is not yet sufficient for our argument but we have to introduce a slightly more involved gate. Assume now that we have two control qubits, c and c', on Alice's side and consider again Bob's qubit as the 
target. We will apply a particular operation which we call a controlled Pauli gate (CP-gate). This gate applies one of the four Pauli-operators on the target qubit depending on the state of the two control qubits and can be written as

$$
\begin{aligned}
U_{C P} & =|00\rangle\langle 00|\otimes \mathbb{1}+| 01\rangle\langle 01| \otimes \sigma_{x} \\
& +|10\rangle\left\langle 10\left|\otimes \sigma_{y}+\right| 11\right\rangle\langle 11| \otimes \sigma_{z},
\end{aligned}
$$

where we have omitted the subscripts $c c^{\prime}$ to make the notation lighter. Given that we are assuming that Alice can teleport any unitary operation to Bob, we can therefore implement a CP-gate between Alice and Bob with Alice acting as the control. We will demonstrate that the CP-gate can be used to establish, starting from a product state between Alice and Bob, a state that contains two shared e-bits. To this end, assume that Bob holds two particles in the maximally entangled state $\left|\phi^{+}\right\rangle_{B}=|00\rangle_{B}+|11\rangle_{B}$ and that Alice holds her two control particles in state $|00\rangle+|01\rangle+|10\rangle+|11\rangle$. The result of the CP operation is

$$
\begin{gathered}
U_{C P}(|00\rangle+|01\rangle+|10\rangle+|11\rangle)_{c c^{\prime}} \otimes(|00\rangle+|11\rangle)_{B}= \\
|00\rangle_{c c^{\prime}}(|00\rangle+|11\rangle)_{B}+|01\rangle_{c c^{\prime}}(|01\rangle+|10\rangle)_{B} \\
+i|10\rangle_{c c^{\prime}}(|01\rangle-|10\rangle)_{B}+|11\rangle_{c c^{\prime}}(|00\rangle-|11\rangle)_{B},
\end{gathered}
$$

which contains 2 e-bits of entanglement shared between Alice and Bob. As entanglement does not increase under LQCC, and the teleportation of $U$ has been done using only LQCC, we conclude that the teleportation of a general $U$ requires at least two e-bits.

Now let us proceed to show that the teleportation of an unknown $U$ also requires the transmission of two classical bits from Alice to Bob. The idea of the proof is to show that per application of the $\mathrm{CP}$ gate Alice can transmit 2 classical bits of information. This implies that the implementation of the $\mathrm{CP}$-gate requires 2 bits of classical communication between Alice and Bob as otherwise we would be able to establish a super-luminal channel between the two parties following an argument analogous to that presented in the original teleportation paper [2]. Imagine the following protocol. Alice encodes four messages in binary notation as $|00\rangle,|01\rangle,|10\rangle,|11\rangle$ in two of her control qubits. Assume that Bob holds two particles in state $\left|\phi^{+}\right\rangle_{B}=|00\rangle+|11\rangle$, as before. The CP-gate is applied between Alice's particle and the first of Bob's particles (using the teleportation procedure of an unknown operation). Depending on the state in which Alice has prepared her two control qubits, Bob will subsequently hold one of the four Bell states, which are mutually orthogonal. Therefore he is able to infer Alice's message and 2 classical bits have been transmitted. As a result, the implementation of the teleportation of an unknown $U$ has to include the transmission of two bits of classical information from Alice to Bob. Consider now the case when the first of Alice's qubits is kept in a fixed state, for instance in state $|0\rangle$. The implementation of a controlled-Pauli operation is now equivalent to implementing a controlled-NOT gate between Alice's second qubit and Bob's qubit [9]. When Alice prepares the state $|+\rangle_{c}=|0\rangle+|1\rangle$, the action of a controlled-NOT gate with Bob qubit being in either state $|+\rangle_{B}$ or in state $|-\rangle_{B}$ is given by

$$
\begin{aligned}
& |+\rangle_{c}|+\rangle_{B} \longmapsto|+\rangle_{c}|+\rangle_{B} \\
& |+\rangle_{c}|-\rangle_{B} \longmapsto|-\rangle_{c}|-\rangle_{B} .
\end{aligned}
$$

Therefore, this operation allows Bob to transmit one bit of information to Alice and, as a consequence, the teleportation of $U$ requires at least one bit of communication from Bob to Alice. Summarizing, the physical principles of nonincrease of entanglement under LQCC and the impossibility of super-luminal communication allow us to establish lower bounds in the resources required for teleporting an unknown quantum operation on a qubit. At least two e-bits of entanglement have to be consumed and, in addition, this quantum channel has to be supplemented by a two way classical communication channel which, in principle, could be non-symmetric. While consistency with causality requires two classical bits being transmitted from Alice to Bob, the lower bound for the amount of classical information transmitted from Bob to Alice has been found to be one bit.

Our main result in [3] was to prove that the transmission of just one classical bit from Bob to Alice is not sufficient if the protocol is meant to work for an arbitrary $U$. We showed that each operation $G_{i}$ necessarily involves a state transfer between the remote parties and therefore, given that quantum state teleportation can be proven to be optimal, the classical communication cost of the remote control process is two bits in each direction. We will analyze now what happens if the requirement of universality is removed and characterize the sets of transformations that can be implemented remotely without resorting to BQST. 


\section{OPTIMAL NON-TRIVIAL PROTOCOL FOR THE IMPLEMENTATION OF AN ARBITRARY $U$}

As explained in detail above, the basic principles establishing the impossibility of superluminal communication and the impossibility of increasing entanglement under LQCC allow us to set the necessary resources for implementing a universal remote control protocol:

- Two shared e-bits between Alice and Bob.

- Two c-bits conveyed from Alice to Bob.

- One c-bit conveyed from Bob to Alice.

We will now show that a protocol can be built which saturates these bounds and achieves $50 \%$ efficiency for the remote implementation of an arbitrary $U$. Given that the optimal protocol consumes two classical bits from Bob to Alice, this is the maximum probability of success if only one bit is conveyed in that direction.

Our starting point can therefore be chosen a pure state of the form

$$
\begin{aligned}
|\chi\rangle_{A B} & =\left|\phi^{+}\right\rangle_{A B} \otimes\left|\phi^{+}\right\rangle_{A B} \otimes|\psi\rangle_{b} \\
& =(|00\rangle+|11\rangle)_{A B} \otimes(|00\rangle+|11\rangle)_{A B} \otimes(\alpha|0\rangle+\beta|1\rangle)_{b}
\end{aligned}
$$

where Alice and Bob share two maximally entangled states and, in addition, Bob holds a qubit in an arbitrary state $|\psi\rangle_{b}=\alpha|0\rangle_{b}+\beta|1\rangle_{b}$. In the following we may omit at times the subscripts referring to the parties $A$ and $B$ to make notation lighter whenever there is no risk of confusion. The aim of the protocol is to end up with Bob holding the transformed state $U|\psi\rangle_{b}$, the operation $U$ being applied only on Alice's side.

\section{A. Local actions on Bob's side}

Let us keep, for the moment, one of the shared e-bits intact. The remaining part of the initial state can be rewritten as

$$
|\lambda\rangle_{A B}=\alpha|0\rangle_{A}|00\rangle_{B}+\beta|0\rangle_{A}|01\rangle_{B}+\alpha|1\rangle_{A}|10\rangle_{B}+\beta|1\rangle_{A}|11\rangle_{B}
$$

where the first qubit belongs to Alice and the other two to Bob. We now perform a controlled-NOT operation on Bob's side using his shared part of the e-bit as a control. After this operation, they share the joint state

$$
|\lambda\rangle_{A B}=\left(\alpha|00\rangle_{A B}+\beta|11\rangle_{A B}\right) \otimes|0\rangle_{B}+\left(\alpha|11\rangle_{A B}+\beta|00\rangle_{A B}\right) \otimes|1\rangle_{B}
$$

Bob now measures his second qubit in the computational basis. If the result is 0 , they do noting, if it is 1 both Alice and Bob perform a spin flip on their qubits. As a result, Alice and Bob now share the partially entangled state

$$
|\psi\rangle_{A B}=\alpha|00\rangle_{A B}+\beta|11\rangle_{A B}
$$

In this way we have managed to make the coefficients $\alpha, \beta$ visible to Alice's side or, in other words, we have distributed the amplitudes $\alpha$ and $\beta$ onto the channel. Note that this part of the protocol has already made use of one e-bit. In addition, one classical bit of information has been conveyed from Bob to Alice.

\section{B. Local actions on Alices's side}

We make now use of the extra e-bit we have kept alone so far. The global state of the system can be written as

$$
\left|\lambda^{\prime}\right\rangle_{A B}=\left(\alpha|00\rangle_{A B}+\beta|11\rangle_{A B}\right) \otimes\left(|00\rangle_{A B}+|11\rangle_{A B}\right)
$$

Alice applies the transformation $U$ to one of her qubits. With this, the global state reads

$$
|\psi\rangle_{A B}=\left(\alpha\left(U|0\rangle_{A}\right)|0\rangle_{B}+\beta\left(U|1\rangle_{A}\right)|1\rangle_{B}\right) \otimes\left(|00\rangle_{A B}+|11\rangle_{A B}\right)
$$


The remaining part of the protocols mimics quantum state teleportation with Alice performing a Bell measurement on her side. This procedure makes use of the extra e-bit and involves the transmission of two classical bits of information from Alice to Bob. We will see in the following that as a result of this protocol, Bob ends up holding a two-qubit state of the form:

$$
(\alpha U|0\rangle+\beta U|1\rangle) \otimes|0\rangle+(\alpha U|0\rangle-\beta U|1\rangle) \otimes|1\rangle=U\left(|\psi\rangle_{b}\right) \otimes|0\rangle+U\left(\sigma_{z}|\psi\rangle_{b}\right) \otimes|1\rangle
$$

A final projective measurement on Bob's side yields the correct transformed state with $50 \%$ probability, the maximum allowed when the transformation $U$ is completely arbitrary and only one bit is conveyed from Alice to Bob.

\section{Detailed steps}

For our purposes, it suffices to parametrize the transformation $U$ as a generic unimodular matrix, i.e. an arbitrary rotation on a qubit of the form

$$
U=\left(\begin{array}{cc}
a & b \\
-b^{*} & a^{*}
\end{array}\right)
$$

where the coefficients $a$ and $b$ obey the unimodular constraint $|a|^{2}+|b|^{2}=1$ Using the Bell basis $\left(\left|\phi^{ \pm}\right\rangle_{A}=|00\rangle_{A} \pm\right.$ $\left.\left.|11\rangle_{A}, \mid \psi^{ \pm}\right)\right\rangle_{A}=|01\rangle_{A} \pm|10\rangle_{A}$ for Alice's qubits, we can rewrite the joint state given by eq. (14) as follows

$$
\begin{aligned}
\left|\lambda^{\prime}\right\rangle_{A B} & =\left|\phi^{+}\right\rangle_{A} \otimes(\alpha|0\rangle U|0\rangle+\beta|1\rangle U|1\rangle) \\
& +\left|\phi^{-}\right\rangle \otimes\left(\mathbb{1} \otimes \sigma_{z}\right)(\alpha|0\rangle U|0\rangle+\beta|1\rangle U|1\rangle) \\
& +\left|\psi^{+}\right\rangle \otimes\left(\mathbb{1} \otimes \sigma_{x}\right)(\alpha|0\rangle U|0\rangle+\beta|1\rangle U|1\rangle) \\
& +\left|\psi^{-}\right\rangle \otimes\left(\mathbb{1} \otimes \sigma_{x} \sigma_{z}\right)(\alpha|0\rangle U|0\rangle+\beta|1\rangle U|1\rangle)
\end{aligned}
$$

Alice now performs a Bell measurement on her two qubits and informs of her results to Bob using a classical channel. Accordingly to Alice's measurement outcomes, Bob performs on his second qubit the same operations as the corresponding to the protocol of quantum state teleportation. As a result of this procedure, he always ends up holding the following two qubit (pure) state

$$
\alpha|0\rangle(a|0\rangle+b|1\rangle)+\beta|1\rangle\left(-b^{*}|0\rangle+a^{*}|1\rangle\right)
$$

, which after a local Hadamard transformation on the first qubit reads

$$
|0\rangle \otimes(\alpha U|0\rangle+\beta U|1\rangle)+|1\rangle \otimes(\alpha U|0\rangle-\beta U|1\rangle)
$$

A final projective measurement on the first qubit leaves Bob holding the correct transformed state by $U$ whenever the measurement outcome is 0 . However, in the case that the local measurement throws the outcome 1 , Bob would hold the wrong state $\alpha U|0\rangle-\beta U|1\rangle$ and, provided that $U$ is completely arbitrary, he cannot restore this state to correct form. As a result, the protocol is successful in $50 \%$ of the cases. Note that this is the maximum efficiency we can expect when only one bit is conveyed from Bob to Alice. It is a remarkable fact, and a direct consequence of the linearity of quantum mechanics [3], that no protocol different from bi-directional quantum state teleportation can achieve the remote implementation of any arbitrary operation on a qubit. But, are there sets of transformations for which is possible for Bob to restore the final state to the correct form $\alpha U|0\rangle+\beta U|1\rangle$ ?

\section{RESTRICTED SET OF OPERATIONS}

As discussed in detail in the previous section, with probability $50 \%$ Bob is left holding the wrong transformed state

$$
\alpha U|0\rangle-\beta U|1\rangle=U \sigma_{z}|\phi\rangle_{B}
$$

Given that the transformation $U$ given by Eq.(16) is completely unknown to him, no subsequent local action can yield the correct transformed state $U|\phi\rangle_{B}$ for every $U$. However, it is clear from the above expression that there are cases 
where implementing a universal correction operation $V$ is possible. Formally, we are seeking for an operator $V$ such that

$$
V U \sigma_{z}|\phi\rangle_{B}=e^{i \delta} U|\phi\rangle_{B}
$$

for any $|\phi\rangle_{B}, \gamma$ being a real parameter. Therefore, the following operator identity must hold

$$
V U=e^{i \delta} U \sigma_{z} .
$$

We can immediately identify a set of transformations that can be remotely implemented. If we set $V=\sigma_{z}$, the two possible uni-modular solutions to Eq.(20) are given by (up to a local change of basis) [10]:

$$
U_{c}=\left(\begin{array}{cc}
a & 0 \\
0 & a^{*}
\end{array}\right)=e^{i \phi \sigma_{z}}
$$

with $a=e^{i \phi}$ that is, the set of operations that commute with $\sigma_{z}$, or transformations of the form

$$
U_{a}=\left(\begin{array}{cc}
0 & b \\
-b^{*} & 0
\end{array}\right)
$$

with $b=e^{i \phi}$ which anticommute with $\sigma_{z}$, i.e., are linear combinations of the Pauli operators $\sigma_{x}$ and $\sigma_{y}$. Any operation within this family can be teleported with $100 \%$ efficiency using a protocol which employs less resources than BQST. We can physically interpret the set of allowed transformations as

- Arbitrary rotations around the z-axis.

- Rotations by $\pi$ around any axis lying within the equatorial plane.

We will illustrate in the next section, using the Bloch sphere representation for qubits, how it can be easily visualized why a universal correction by means of the application of the operator $\sigma_{z}$ is possible is these cases.

There is still a question that remains to be addressed. Are the sets of operations we have just described the only ones that can be implemented remotely by non-trivial means? We will postpone the issue of uniqueness till Section VI.

\section{GEOMETRICAL INTERPRETATION}

The aim of this section is just to provide an intuitive geometrical picture in order to visualize which transformations can be implemented remotely by non-trivial means and illustrate the role of the final restoration step on Bob's side. Let us consider first a very simple scenario in which Bob is holding a qubit state lying in the equatorial plane of the Bloch sphere,

$$
|\phi\rangle_{b}=\alpha|0\rangle+\beta|1\rangle=\frac{1}{\sqrt{2}}\left(|0\rangle+e^{i \zeta}|1\rangle\right)
$$

Imagine now that the transformation we want to implement remotely is just a spin flip, i.e., $U=\sigma_{x}$ (Obviously Alice does not know this!). In this case, given that the Pauli operator anticommute with $\sigma_{z}$, the protocol described in the previous section will result in Bob to hold the correct transformed state $\sigma_{x}|\phi\rangle_{B}$. If Bob follows the prescribed rules, prior to the final correction step with $50 \%$ probability he holds the correct transformed state and with $50 \%$ probability he holds the erroneous state

$$
|\psi\rangle_{b, W}=\alpha U|0\rangle-\beta U|1\rangle=U\left(\frac{1}{\sqrt{2}}\left(|0\rangle-e^{i \zeta}|1\rangle\right) .\right.
$$

Therefore, we can also consider the wrong transformed state as the transformed by $U$ of the qubit state $|\bar{\psi}\rangle_{b}=\sigma_{z}|\psi\rangle_{b}$. Which state Bob ends up holding depends on certain measurement outcome and therefore he knows whether a subsequent correction step is necessary or not. The relative position of the Bloch vectors representing the initial states $|\psi\rangle_{B}$ and $|\bar{\psi}\rangle_{B}$ and their transformed vectors by $U$ are shown in figure 1. In this case, states $|\psi\rangle_{B}$ and $|\bar{\psi}\rangle_{B}$ are orthogonal and their associated Bloch vectors lie opposite in the equatorial plane of the Bloch sphere. The action of $U$ preserves the relative orientation and the Bloch vectors associated with the transformed states by $\mathrm{U}$, dashed lines in the figure, are opposite as well. The key point is that a subsequent application of the operation $\sigma_{z}$ onto 
the wrong transformed state just flips its Bloch vector and yields the correct state. These considerations may sound rather trivial but it is all we need to intuitively understand how the protocol works in the general case.

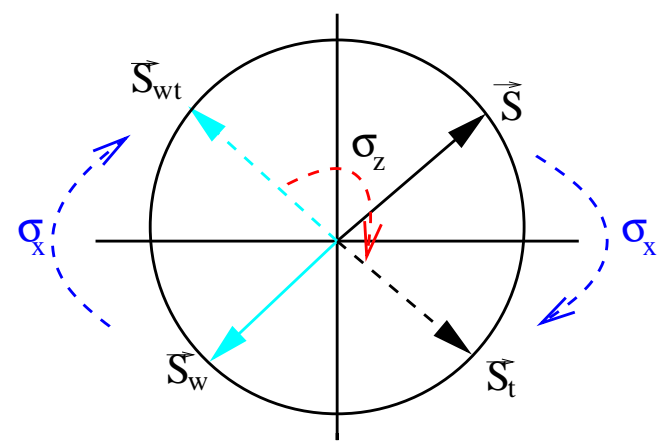

FIG. 1. Geometrical interpretation of the restoration to the correct transformed state when the transformation $U$ belongs to a restricted set. See the text for details.

Imagine now that the transformations $U$ is not simply a Pauli operator but a transformation of the general form given by eq.(16). Assuming that Bobs state lies initially in the equatorial plane, as before, the corresponding Bloch vector of the transformed state by $U$ does not longer lie onto the equator of the Bloch sphere and has in general a non-zero z-component $S_{z}=|\alpha|^{2}-|\beta|^{2}$ (we have defined $S_{i}=\operatorname{tr} \rho \sigma_{i}$ ) However, this component is equal to zero if the transformation $U$ either commutes or anticommutes with $\sigma_{z}$ (operations of the form $U_{c}$ or $U_{a}$ ). In this case, we recover the situation discussed before. The transformed Bloch vectors lie opposite along some direction contained in the equatorial plane and a final step via the application of $\sigma_{z}$ restores the wrong transformed state to the correct one. What happens in general? The easiest way to analyze the general case, where Bob holds an arbitrary qubit state, is to parametrize it as a generic spinor and split the representation in terms of the associated Bloch vectors into two components as follows

$$
\rho_{b}=|\psi\rangle\langle\psi|=\frac{1}{2}\left(\mathbb{1}+S_{x} \sigma_{x}+S_{y} \sigma_{y}+S_{z} \sigma_{z}\right) .
$$

Analogously, the wrong transformed state can be thought of as obtained from $U$ acting upon the state

$$
\bar{\rho}_{b}=|\bar{\psi}\rangle\langle\bar{\psi}|=\frac{1}{2}\left(\mathbb{1}-S_{x} \sigma_{x}-S_{y} \sigma_{y}+S_{z} \sigma_{z}\right),
$$

so we can write the erroneous transformed state as

$$
U \bar{\rho}_{b} U^{\dagger}=\frac{1}{2}\left(\mathbb{1}-U\left(S_{x} \sigma_{x}+S_{y} \sigma_{y}\right) U^{\dagger}+U S_{z} \sigma_{z} U^{\dagger}\right)
$$

Consider the case where the transformation $U$ commutes with the action of $\sigma_{z}$. When Bob applies the final correction step, the transformed state reads

$$
\begin{aligned}
\sigma_{z} U \bar{\rho}_{b} U^{\dagger} \sigma_{z} & =\frac{1}{2}\left(\mathbb{1}-\sigma_{z} U\left(S_{x} \sigma_{x}+S_{y} \sigma_{y}\right) U^{\dagger} \sigma_{z}+\sigma_{z} U S_{z} \sigma_{z} U^{\dagger} \sigma_{z}\right) \\
& =\frac{1}{2}\left(\mathbb{1}+U\left(S_{x} \sigma_{x}+S_{y} \sigma_{y}\right) U^{\dagger}+U S_{z} \sigma_{z} U^{\dagger}\right)=U \rho_{B} U^{\dagger},
\end{aligned}
$$

where we have taken into account that Pauli operators anti-commute among themselves and that $\sigma^{2}=\mathbb{1}$. A similar argument holds for the case where $U$ anti-commutes with $\sigma_{z}$. Resuming our geometrical picture, in the general case the corresponding Bloch vectors associated to the states $|\psi\rangle_{b}$ and $|\bar{\psi}\rangle_{b}$ have the same z-component while the corresponding projections onto the equatorial plane lie opposite. Therefore, under the action of a transformation $U$ which either commutes or anticommutes with $\sigma_{z}$, we recover the situation discussed at the beginning of this section and a final correction by means of applying the operation $\sigma_{z}$ restores the correct transformed state.

\section{CHARACTERIZATION OF SETS THAT ALLOW REMOTE IMPLEMENTATION WITHOUT BIDIRECTIONAL STATE TELEPORTATION}

So far we have identified two sets of transformations that can be implemented remotely without resorting to BQST (bidirectional state teleportation). However, the procedure by which they have been identified does not allow to draw 
any conclusion as far as their uniqueness is concerned. This is the aim of this section. To do this we first establish necessary conditions for avoiding BQST and then we show the uniqueness of the two sets of transformations.

\section{A. Necessary conditions for avoiding BQST}

Let the set of operators that can be remotely implemented on Bob's qubit be labeled as $\mathcal{U}$. We know [3] that if $\mathcal{U}$ is the full set of unimodular operations on a qubit, the protocol necessarily teleports the state of Bob's qubit to Alice, that is, every state undergoes BQST. In contrast, in the protocol described in Section III, where $\mathcal{U}$ contains operators of the form Eqs. (21) and (22), it is easy to show that only the two orthogonal states $|0\rangle$ and $|1\rangle$ undergo BQST. In this section we examine the relationship between the size of the set $\mathcal{U}$ and the number of states that undergo BQST. From this we show that if Bob is restricted to sending $1 \mathrm{c}$-bit to Alice, then the set $\mathcal{U}$ comprises two particular subsets.

\section{Subsets of operators}

In [3] we showed that the if the teleported operation $U$ is arbitrary, that is $\mathcal{U}$ is the full set of unimodular operations, the final state of the ancilla is independent of $U$. However, here the set of operators is restricted and so the final state of the ancilla may depend on which operation is teleported. Hence we reexpress the operation of the black box as (cf Eq. (11))

$$
G_{2} U_{n} G_{1}\left(|\chi\rangle_{a A B}|\psi\rangle_{b}\right)=\left|\Phi\left(\chi, U_{n}\right)\right\rangle_{a A B} U_{n}|\psi\rangle_{b}
$$

for $U_{n} \in \mathcal{U}$. We know that the final state $\left|\Phi\left(\chi, U_{n}\right)\right\rangle_{a A B}$ is independent of $|\psi\rangle_{b}$ by the same arguments presented in our previous work [3].

Consider action of the gate for a linear combination of operators $U=\sum_{n} c_{n} U_{n}$ where $U, U_{n} \in \mathcal{U}$ :

$$
\begin{aligned}
G_{2} U_{n} G_{1}|\chi\rangle_{a A B}|\psi\rangle_{b} & =\sum_{n} c_{n} G_{2} U_{n} G_{1}|\chi\rangle_{a A B}|\psi\rangle_{b} \\
& =\sum_{n} c_{n}\left|\Phi\left(\chi, U_{n}\right)\right\rangle_{a A B} U_{n}|\psi\rangle_{b}
\end{aligned}
$$

which equals $|\Phi(\chi, U)\rangle_{a A B} U|\psi\rangle_{b}$ only if $\left|\Phi\left(\chi, U_{n}\right)\right\rangle_{a A B}$ is independent of $U_{n}$. In other words, linearly dependent operators share the same final state. This final state may depend on set of linearly dependent control operators, however. Indeed, we subdivide the set $\mathcal{U}$ into subsets

$$
\mathcal{U}=\mathcal{U}^{(1)} \cup \mathcal{U}^{(2)} \cup \ldots
$$

which leave the state of the ancilla in the same final state:

$$
\left|\Phi\left(\chi, U_{i}^{(n)}\right)\right\rangle=\left|\Phi\left(\chi, U_{j}^{(n)}\right)\right\rangle=\left|\Phi^{(n)}(\chi)\right\rangle
$$

where $U_{i}^{(n)} \in \mathcal{U}^{(n)}$. [We use the superscript " $(n)$ " to label a subset and its elements.] It follows that the subsets $\mathcal{U}^{(n)}$ are linearly independent in the sense that an operator in one subset cannot be written as a linear combination of operators from other sets. Also, the subsets are clearly disjoint as each operator $U \in \mathcal{U}$ belongs to one and only one subset $\mathcal{U}^{(n)}$. Since there are a maximum of 4 linearly independent operators on the 2 dimensional state space, there are thus a maximum of 4 subsets $\mathcal{U}^{(n)} \subset \mathcal{U}$.

\section{Special case $G_{1}=\mathbb{1}$}

It is interesting to consider the special case where $G_{1}=\mathbb{1}$. We now show that for this case there are a maximum of 4 operators which can be teleported. Consider two operators, $U_{1}^{(n)}$ and $U_{2}^{(m)}$ and choose an orthogonal pair of states $\left\langle\psi \mid \psi_{\perp}\right\rangle=0$ such that

$$
\begin{aligned}
U_{1}^{(n)}|\psi\rangle & =|\phi\rangle, \\
U_{2}^{(m)}\left|\psi_{\perp}\right\rangle & =\left|\phi^{\prime}\right\rangle
\end{aligned}
$$


where $\left\langle\phi \mid \phi^{\prime}\right\rangle \neq 0$ and $U_{i}^{(k)} \in \mathcal{U}^{(k)}$. The fact that this is possible is proved in the Appendix. Thus, we can write

$$
\begin{aligned}
U_{1}^{(n)}|\chi\rangle_{a A B}|\psi\rangle_{b} & =G_{2}^{\dagger}\left|\Phi^{(n)}(\chi)\right\rangle_{a A B} U_{1}^{(n)}|\psi\rangle_{b} \\
& =G_{2}^{\dagger}\left|\Phi^{(n)}(\chi)\right\rangle_{a A B}|\phi\rangle_{b} \\
U_{2}^{(m)}|\chi\rangle_{a A B}\left|\psi_{\perp}\right\rangle_{b} & =G_{2}^{\dagger}\left|\Phi^{(m)}(\chi)\right\rangle_{a A B} U_{1}^{(m)}\left|\psi_{\perp}\right\rangle_{b} \\
& =G_{2}^{\dagger}\left|\Phi^{(m)}(\chi)\right\rangle_{a A B}\left|\phi^{\prime}\right\rangle_{b}
\end{aligned}
$$

The inner product of the left-hand sides of Eqs. (32) and (33) is zero and so

$$
0=\left\langle\Phi^{(n)}(\chi) \mid \Phi^{(m)}(\chi)\right\rangle_{a A B}\left\langle\phi \mid \phi^{\prime}\right\rangle_{b} .
$$

But if $n=m$, then $\left\langle\Phi^{(n)}(\chi) \mid \Phi^{(m)}(\chi)\right\rangle_{a A B}=1$ and Eq. (34) cannot be satisfied. We conclude that each subset contains only one operator.

Also, if $n \neq m$ (i.e. different subsets) then Eq. (34) implies $\left\langle\Phi^{(n)}(\chi) \mid \Phi^{(m)}(\chi)\right\rangle_{a A B}=0$ and so the final ancilla states are orthogonal. The number of operators able to be teleported therefore depends on the dimension of the ancilla state space. Provided this can be made large enough, there will be a maximum of 4 operators able to be teleported with $G_{1}=\mathbb{1}$ (because there are a maximum of 4 linearly-independent subsets).

The fact that the final states of the ancilla are orthogonal for different operators means that the operators themselves are orthogonal. Imagine that Alice has a son called Bobby in her lab. She teleports the operator to Bobby and together they examine the state of the final state of their (local) ancilla. From this they can determine which operator Alice teleported. Alice can communicate this to Bob using 2 classical bits of information, and Bob can then carry out locally the corresponding operation on his qubit.

Hence the special case $G_{1}=\mathbb{1}$ leads to a trivial classical remote control scenario. For the remainder of this paper we only consider the case where $G_{1} \neq \mathbb{1}$.

\section{Conditions for the BQST of a state}

We now look at a sufficient condition on the set $\mathcal{U}$ for the BQST of a state. This will give us a necessary condition for avoiding BQST for a set of states. Choose $U^{(n)} \in \mathcal{U}^{(n)}$ and let

$$
U^{(n)}|\psi\rangle=|\phi\rangle .
$$

Thus we have

$$
G_{2} U^{(n)} G_{1}|\chi\rangle_{a A B}|\psi\rangle_{b}=\left|\Phi^{(n)}(\chi)\right\rangle_{a A B} U^{(n)}|\psi\rangle_{b}=\left|\Phi^{(n)}(\chi)\right\rangle_{a A B}|\phi\rangle_{b}
$$

and so

$$
G_{1}|\chi\rangle_{a A B}|\psi\rangle_{b}=\left[U^{(n)}\right]^{\dagger} G_{2}^{\dagger}\left|\Phi^{(n)}(\chi)\right\rangle_{a A B}|\phi\rangle_{b}
$$

Next we construct the unimodular operator $Q(\alpha,|\xi\rangle)$ as follows

$$
Q(\alpha,|\xi\rangle) \equiv e^{i \alpha}|\xi\rangle\langle\xi|+e^{-i \alpha}(\mathbb{1}-|\xi\rangle\langle\xi|)
$$

for $\alpha \neq 0, \pi, 2 \pi, \ldots$ and arbitrary (normalised) state $|\xi\rangle$. This operator has the property that

$$
Q(\alpha,|\phi\rangle) U^{(n)}=U^{(n)} Q(\alpha,|\psi\rangle) .
$$

If $U^{(n)} Q(\alpha,|\psi\rangle) \in \mathcal{U}^{(n)}$ then we can replace $U^{(n)}$ in Eq. (36) with $U^{(n)} Q(\alpha,|\psi\rangle)$ and obtain from Eq. (37)

$$
\begin{aligned}
Q\left(\alpha,|\phi\rangle_{a}\right) G_{1}|\chi\rangle_{a A B}|\psi\rangle_{b} & =\left[U^{(n)}\right]^{\dagger} G_{2}^{\dagger}\left|\Phi^{(n)}(\chi)\right\rangle_{a A B} U^{(n)} Q\left(\alpha,|\psi\rangle_{b}\right)|\psi\rangle_{b} \\
& =e^{i \alpha}\left[U^{(n)}\right]^{\dagger} G_{2}^{\dagger}\left|\Phi^{(n)}(\chi)\right\rangle_{a A B}|\phi\rangle_{b} .
\end{aligned}
$$

Comparing Eq. (37) with Eq. (39) shows that $G_{1}|\chi\rangle_{a A B}|\psi\rangle_{b}$ is an eigenstate of $Q\left(\alpha,|\psi\rangle_{a}\right)$, i.e.

$$
G_{1}|\chi\rangle_{a A B}|\psi\rangle_{b}=|\psi\rangle_{a} \otimes \ldots
$$


or, in other words, that the state of Bob's qubit is necessarily teleported to Alice by the operation of $G_{1}$. Note that if $U^{(n)} Q(\alpha,|\psi\rangle)$ belongs to a different subset, say $\mathcal{U}^{(m)}$ with $m \neq n$, then instead of Eq. (39) we get

$$
\begin{aligned}
Q\left(\alpha,|\phi\rangle_{a}\right) G_{1}|\chi\rangle_{a A B}|\psi\rangle_{b} & =\left[U^{(n)}\right]^{\dagger} G_{2}^{\dagger}\left|\Phi^{(m)}(\chi)\right\rangle_{a A B} U^{(n)} Q\left(\alpha,|\psi\rangle_{b}\right)|\psi\rangle_{b} \\
& =e^{i \alpha}\left[U^{(n)}\right]^{\dagger} G_{2}^{\dagger}\left|\Phi^{(m)}(\chi)\right\rangle_{a A B}|\phi\rangle_{b} \\
& \neq e^{i \alpha}\left[U^{(n)}\right]^{\dagger} G_{2}^{\dagger}\left|\Phi^{(n)}(\chi)\right\rangle_{a A B}|\phi\rangle_{b}
\end{aligned}
$$

and so the state of Bob's qubit is not teleported by $G_{1}$ to the qubit operated on by $U^{(n)}$. Hence we can state a sufficient condition for BQST as follows: BQST occurs for a state $|\psi\rangle$ when at least one value of $\alpha \neq 0, \pi, 2 \pi, \ldots$ can be found such that $U^{(n)} Q(\alpha,|\psi\rangle) \in \mathcal{U}^{(n)}$ for at least one operator $U^{(n)} \in \mathcal{U}^{(n)}$ for any $\mathcal{U}^{(n)} \subset \mathcal{U}$.

Consider, for the moment, the case where we insist that none of the states $|\psi\rangle$ undergo BQST. This requires that $U^{(n)} Q(\alpha,|\psi\rangle) \notin \mathcal{U}^{(n)}$ for all $|\psi\rangle$, all $U^{(n)} \in \mathcal{U}^{(n)}$, all $\mathcal{U}^{(n)} \in \mathcal{U}$ and all $\alpha \neq 0, \pi, 2 \pi, \ldots$ The set of $Q(\alpha,|\psi\rangle)$ for all $|\psi\rangle$ and all $\alpha \neq 0, \pi, 2 \pi, \ldots$ is the set of all unimodular operators minus the the set of operators which are proportional to the identity. Assume for the moment that $\mathcal{U}^{(n)}$ contains the two operators $U_{1}^{(n)}, U_{2}^{(n)}$ where $U_{1}^{(n)} \neq e^{i \theta} U_{2}^{(n)}$ for any real $\theta$. We can set $Q(\alpha,|\psi\rangle)=\left[U_{1}^{(n)}\right]^{\dagger} U_{2}$ for an appropriate choice of $|\psi\rangle$ and $\alpha$, and so $U_{1}^{(n)} Q(\alpha,|\psi\rangle)=U_{2}^{(n)} \in \mathcal{U}^{(n)}$. This means that the state $|\psi\rangle$ would be BQST contradicting our starting point. Clearly if no states are to undergo BQST then each subset $\mathcal{U}^{(n)}$ cannot contain more than one operator (up to an imaginary phase factor). Hence, for the case where no states are BQST, $\mathcal{U}$ contains at most 4 linearly independent operators. We note that if the 4 operators are orthogonal (i.e. related to the identity operator and 4 Pauli operators by a fixed transformation) Alice may distinguish between them using local means and thus send 2 classical bits to Bob who could then implement locally the appropriate operation on his qubit. The general case, however, would require either more measurements by Alice to determine the operator, or a more sophisticated channel between Alice and Bob (i.e. with shared ebits etc.)

Returning to the more general case, one can see from Eq. (38) that $Q(\alpha,|\psi\rangle)=Q\left(-\alpha,\left|\psi_{\perp}\right\rangle\right)$ where $\left\langle\psi_{\perp} \mid \psi\right\rangle=0$ and so if $|\psi\rangle$ undergoes BQST then so to are the states orthogonal to $|\psi\rangle$. Non-trivial remote control therefore necessarily incurs BQST for at least one pair of orthogonal states. Bob can communicate 1 classical bit to Alice by preparing his qubit in one of these orthogonal states and stopping the protocol after $G_{1}$. The scheme we are most interested in is where Bob sends exactly 1 classical bit of information to Alice. Henceforth we only consider the case where exactly one pair of orthogonal states undergo BQST with all other states avoiding BQST.

\section{BQST of a single pair of states}

For brevity we take the pair of orthogonal states that are BQST to be the computational basis states: $|0\rangle$, $|1\rangle$. (It is straight forward to generalize our analysis to an arbitrary pair.) All other states,

$$
\left|\psi^{\prime}\right\rangle=a|0\rangle+b|1\rangle
$$

for $a, b \neq 0,1$, do not undergo BQST. We can write this as

$$
U_{i}^{(n)} Q\left(\alpha,\left|\psi^{\prime}\right\rangle\right) \notin \mathcal{U}^{(n)}
$$

or, equivalently,

$$
Q\left(\alpha,\left|\psi^{\prime}\right\rangle\right) \notin\left[U_{i}^{(n)}\right]^{\dagger} \mathcal{U}^{(n)}
$$

for all $\alpha \neq 0, \pi, 2 \pi, \ldots$, all $\left|\psi^{\prime}\right\rangle \neq|0\rangle,|1\rangle$, all $U_{i}^{(n)} \in \mathcal{U}^{(n)}$ and all subsets $\mathcal{U}^{(n)} \subset \mathcal{U}$. The set of operators $\left\{Q\left(\alpha,\left|\psi^{\prime}\right\rangle\right)\right\}$ here is the set of all unimodular operators not diagonalized by $|0\rangle,|1\rangle$. Hence each set $\left[U_{i}^{(n)}\right]^{\dagger} \mathcal{U}^{(n)}$ contains operators which are diagonalized by $|0\rangle,|1\rangle$. Thus all elements of each subset $\mathcal{U}^{(n)} \subset \mathcal{U}$ have the form

$$
\begin{aligned}
U_{\beta}^{(n)} & =U_{0}^{(n)}\left(e^{i \beta}|0\rangle\left\langle 0\left|+e^{-i \beta}\right| 1\right\rangle\langle 1|\right) \\
& =U_{0}^{(n)} Q(\beta,|0\rangle) \\
& =U_{0}^{(n)} e^{i \beta \sigma_{z}} .
\end{aligned}
$$


If the subsets $\mathcal{U}^{(n)}$ are the largest possible (i.e. $\mathcal{U}^{(n)}$ contains the operators $U_{\beta}^{(n)}$ for all $\beta$ ) then there are a maximum of 2 subsets $\mathcal{U}^{(n)} \subset \mathcal{U}$. To see this consider an arbitrary, unimodular, linear combination of the elements of 2 subsets $\mathcal{U}^{(1)}$ and $\mathcal{U}^{(2)}$ :

$$
U=x U_{0}^{(1)} e^{i \beta \sigma_{z}}+y U_{0}^{(2)} e^{i \gamma \sigma_{z}}
$$

where $x$ and $y$ are real numbers. We can write this as

$$
\left[U_{0}^{(1)}\right]^{\dagger} U=x e^{i \beta \sigma_{z}}+y\left[U_{0}^{(1)}\right]^{\dagger} U_{0}^{(2)} e^{i \gamma \sigma_{z}}
$$

or, in matrix form, as

$$
\left[\begin{array}{cc}
c & d \\
-d^{*} & c^{*}
\end{array}\right]=x\left[\begin{array}{cc}
e^{i \beta} & 0 \\
0 & e^{-i \beta}
\end{array}\right]+y\left[\begin{array}{cc}
a e^{i \gamma} & b e^{-i \gamma} \\
-b^{*} e^{i \gamma} & a^{*} e^{-i \gamma}
\end{array}\right]
$$

where

$$
\begin{gathered}
{\left[U_{0}^{(1)}\right]^{\dagger} U=\left[\begin{array}{cc}
c & d \\
-d^{*} & c^{*}
\end{array}\right]} \\
{\left[U_{0}^{(1)}\right]^{\dagger} U_{0}^{(2)}=\left[\begin{array}{cc}
a & b \\
-b^{*} & a^{*}
\end{array}\right] .}
\end{gathered}
$$

Clearly, $y e^{-i \gamma}=d / b$ and $x e^{i \beta}=c-y a e^{i \gamma}$ which can be solved for real values of $x, y, \beta$ and $\gamma$ for arbitrary $c$ and $d$ satisfying $|c|^{2}+|d|^{2}=1$. This shows that every unimodular operator $U^{\prime}=\left[U_{0}^{(1)}\right]^{\dagger} U$, and hence every unimodular operator $U=U_{0}^{(1)} U^{\prime}$, can be written in terms of a linear combination of operators in the subsets $\mathcal{U}^{(1)}$ and $\mathcal{U}^{(2)}$. These two subsets are, therefore, the only linearly independent subsets.

We note that restricting the number of subsets to 1 and choosing either $U_{0}^{(1)}=\mathbb{1}$ or $U_{0}^{(1)}=i \sigma_{y}$ in Eq. (40) corresponds to the situation in the $1-1-1$ protocol. On the other hand choosing $U_{0}^{(1)}=\mathbb{1}$ and $U_{0}^{(2)}=i \sigma_{y}$ corresponds to the situation in the $2-2-1$ protocol.

To sum up this subsection: to avoid BQST for all states, the set of control operators must be restricted to a set of a maximum of 4 linearly independent operators; if one state undergoes BQST then so are the states orthogonal to it; if Bob is restricted to sending 1 classical bit to Alice then only 1 pair of orthogonal states can undergo BQST and the set of control operations $\mathcal{U}$ can be divided into a maximum of 4 subsets $\mathcal{U}^{(n)} \subset \mathcal{U}$ whose elements have the form Eq. (40); if the subsets $\mathcal{U}^{(n)}$ in Eq. (40) contain operators $U_{\beta}^{(n)}$ for all $\beta$ then only 2 subsets are possible.

Finally, we note that these conditions on the set $\mathcal{U}$ of controlled operators are necessary for avoiding the BQST of various states. They are not sufficient conditions because $G_{1}$ and $G_{2}$ can be chosen to perform BQST for all states, irrespective of the restrictions on $\mathcal{U}$.

\section{B. Full characterization of classes of operators allowing for non-trivial remote implementation.}

In this subsection we now wish to complete the characterization of the classes of state that can be implemented without BQST. We will show that a protocol that consumes 2 shared ebits +2 bit of classical communication from $\mathrm{A} \rightarrow \mathrm{B}+1$ bit of classical communication from $\mathrm{B} \rightarrow \mathrm{A}(221)$ for teleportation of unitary operations is only possible when the operations are drawn from the following two sets:

$$
\begin{aligned}
& \text { Set A : }\left[\left(\begin{array}{cc}
e^{i \phi} & 0 \\
0 & e^{-i \phi}
\end{array}\right), \phi \in \mathbb{R}\right] \\
& \text { Set B : }\left[\left(\begin{array}{cc}
a & b \\
-b^{*} & a^{*}
\end{array}\right)\left(\begin{array}{cc}
e^{i \phi} & 0 \\
0 & e^{-i \phi}
\end{array}\right), \phi \in \mathbb{R}\right]
\end{aligned}
$$

under the constraint that either $|a|=1$ (trivial) or $|b|=1$. Any other choices will require more resources \. Together with the results from the previous section this then concludes our characterization of those operations that allow for non-trivial remote implementation.

\footnotetext{
${ }^{1}$ Of course we have in addition the freedom of choice of basis, ie we can change all the above sets jointly by a fixed basis change, but that is a trivial freedom
} 
As outlined in section II and used throughout this paper the most general of any possible protocol is given by

$$
G_{2} U G_{1}|\chi\rangle_{A}|\psi\rangle_{B}=|\bar{\chi}\rangle(U|\psi\rangle)_{B}
$$

where without loss of generality the state $|\chi\rangle$ is a tensor product state. For whatever form of $G_{1}$ we can always write Eq. 43 as

$$
G_{2} U G_{1}|\chi\rangle_{A}|\psi\rangle_{B}=G_{2}\left(\left(U|0\rangle_{A}\right)\left|\Phi_{0}\right\rangle_{A B}+\left(U|1\rangle_{A}\right)\left|\Phi_{1}\right\rangle_{A B}\right)=|\bar{\chi}\rangle_{A B}(U|\psi\rangle)_{B}
$$

We can always write $|\psi\rangle=\alpha|0\rangle+\beta|1\rangle$ and note that $|\bar{\chi}\rangle$ is independent from both $\phi$ and $|\psi\rangle$, but may of course depend on $a$ and $b$. From normalization we have $\left\langle\Phi_{0} \mid \Phi_{0}\right\rangle+\left\langle\Phi_{1} \mid \Phi_{1}\right\rangle=1$. If we now evaluate eq. 44 for two unitaries $U_{1}, U_{2}$ from the above sets Eqs. 41,42 we can obtain the following scalar product

$$
\sum_{i j}\left\langle i\left|U_{2}^{\dagger} U_{1}\right| j\right\rangle\left\langle\Phi_{i} \mid \Phi_{j}\right\rangle=\left(|\alpha|^{2}\left\langle 0\left|U_{2}^{\dagger} U_{1}\right| 0\right\rangle+|\beta|^{2}\left\langle 1\left|U_{2}^{\dagger} U_{1}\right| 1\right\rangle+\alpha \beta^{*}\left\langle 1\left|U_{2}^{\dagger} U_{1}\right| 0\right\rangle+\alpha^{*} \beta\left\langle 0\left|U_{2}^{\dagger} U_{1}\right| 1\right\rangle\right)\left\langle\bar{\chi}_{2} \mid \bar{\chi}_{1}\right\rangle .
$$

The proof proceeds in essentially two steps. First we will demonstrate that in the protocol the operation $G_{1}$ will generally create an entangled state between the qubit $U$ is acting upon and the rest of the systems. Up to local rotations any entangled state is of the form $r|00\rangle+s|11\rangle$. In the basis where the entangled state can be written like this we will then show, that when $U$ acts on it we can only find a $G_{2}$ that recovers $U|\psi\rangle$ if either $|a|=1$ or $|b|=1$. This then concludes the proof.

i) Assume that there is no entanglement generated by $G_{1}$.

Given that the set of transformations that we want to teleport is non-trivial, ie they are generally non-orthogonal, the transformation $G_{1}$ has to be non-trivial. This implies in particular that a strategy of distinguishing the unitaries is not possible. Therefore we cannot have the situation that $\left|\Phi_{0}\right\rangle=\left|\Phi_{1}\right\rangle=|\psi\rangle$ for all unitaries $U$. 2

Now let us assume that $G_{1}$ does not generate an entangled state which requires that

$$
\left|\Phi_{0}\right\rangle=\frac{x^{\prime}}{y^{\prime}}\left|\Phi_{1}\right\rangle
$$

Under this assumption we will now demonstrate that then $x^{\prime} / y^{\prime}=\alpha / \beta$. To this end let us make the special choice $U_{2}=1$ which simplifies the analysis and is sufficient to generate the desired result. Then we have

$$
\begin{aligned}
& \left\langle 0\left|U_{1}\right| 0\right\rangle\left|x^{\prime}\right|^{2}+\left\langle 1\left|U_{1}\right| 1\right\rangle\left|y^{\prime}\right|^{2}+\left\langle 0\left|U_{1}\right| 1\right\rangle\left(x^{\prime}\right)^{*} y^{\prime}+\left\langle 1\left|U_{1}\right| 0\right\rangle x^{\prime}\left(y^{\prime}\right)^{*}= \\
& \left(|\alpha|^{2}\left\langle 0\left|U_{1}\right| 0\right\rangle+|\beta|^{2}\left\langle 1\left|U_{1}\right| 1\right\rangle+\alpha \beta^{*}\left\langle 1\left|U_{1}\right| 0\right\rangle+\alpha^{*} \beta\left\langle 0\left|U_{1}\right| 1\right\rangle\right) g
\end{aligned}
$$

where $g$ depends on whether $U_{1}$ is chosen from set $\mathrm{A}(g=1)$ or set B ( $g$ to be determined in a moment). Therefore

$$
\left\langle 0\left|U_{1}\right| 0\right\rangle\left(\left|x^{\prime}\right|^{2}-g|\alpha|^{2}\right)+\left\langle 1\left|U_{1}\right| 1\right\rangle\left(\left|y^{\prime}\right|^{2}-g|\beta|^{2}\right)+\left\langle 0\left|U_{1}\right| 1\right\rangle\left(\left(x^{\prime}\right)^{*} y^{\prime}-g \alpha^{*} \beta\right)+\left\langle 1\left|U_{1}\right| 0\right\rangle\left(x^{\prime}\left(y^{\prime}\right)^{*}-g \alpha \beta^{*}\right)=0
$$

If we chose

$$
\left(\begin{array}{cc}
e^{i \phi} & 0 \\
0 & e^{-i \phi}
\end{array}\right)
$$

with $\phi=0$ and $\phi=\pi / 2$ (which means $g=1$ ) then we get two equations and with the resulting condition

$$
\left|x^{\prime}\right|=|\alpha| \text { and }\left|y^{\prime}\right|=|\beta|
$$

Now we chose two matrices from the set B to determine $g$. From equation (48) we then find that

$$
\begin{aligned}
& a^{*}(1-g)|\beta|^{2}+b\left(x^{\prime *} y^{\prime}-\alpha^{*} \beta g\right)=0 \\
& a(1-g)|\alpha|^{2}-b^{*}\left(x^{\prime} y^{\prime *}-\alpha \beta^{*} g\right)=0
\end{aligned}
$$

\footnotetext{
${ }^{2}$ This remark is relevant because for the case of four orthogonal transformations the following argument does not hold, because we assume that $|\chi\rangle$ is independent of $U$ which only needs to hold when one wishes to teleport non-orthogonal transformations!
} 
as coefficients in front of $e^{i \phi}$ and $e^{-i \phi}$ have to vanish. As $a, b$ and $g$ are fixed we can now only vary $\alpha$ and $\beta$. We know that $|\bar{\chi}\rangle$ and therefore $g$ do not depend on the choice of $\alpha$ and $\beta$. To determine $g$ let us now chose a special case, namely $\alpha=0$ :

In that case we see from (52) that

$$
b^{*} x^{\prime} y^{\prime *}=0
$$

and therefore from (51) we find

$$
a^{*}(1-g)=0
$$

Now we can consider three cases:

a) $a \neq 0, b \neq 0$ : Then $g=1$.

b) $|a|=1$ : Then $g=0$, but in that case the sets A and B are identical and we already know the optimal protocol.

c) $|b|=1$ : Again we know the optimal protocol already.

Therefore, we only need to consider the case where $|g|=1$ and $a \neq 0, b \neq 0$. Then we have that $x^{\prime *} y^{\prime}=\alpha^{*} \beta$. Dividing both sides by $\left|x^{\prime}\right|^{2}$ gives

$$
\frac{y^{\prime}}{x^{\prime}}=\frac{\alpha^{*} \beta}{\left|x^{\prime}\right|^{2}}=\frac{\alpha^{*} \beta}{|\alpha|^{2}}=\frac{\beta}{\alpha}
$$

This implies

$$
|0\rangle\left|\Phi_{0}\right\rangle+|1\rangle\left|\Phi_{1}\right\rangle=\frac{1}{\beta}(\alpha|0\rangle+\beta|1\rangle)\left|\Phi_{1}\right\rangle
$$

As the state $|\psi\rangle$ is general, this implies that $G_{1}$ is a state transfer from Bob to Alice and the resource cost is 2 bits from Bob to Alice. if we only wish to expend 1 bit from Bob to Alice, then this is not a valid option and we can then therefore say that in general $G_{1}$ will produce an entangled state.

ii) Now we can assume that there is a state $|\psi\rangle$ such that $G_{1}$ acting on $|\chi\rangle\left|\psi_{1}\right\rangle$ generates an entangled state. Let us now make a basis change such that we can write

$$
G_{1}|\chi\rangle\left|\psi_{1}\right\rangle=r|0\rangle|0\rangle+s|1\rangle|1\rangle
$$

Now we have to show that when a $U$ from any of the sets A or B acts on one half of the state (57), it is not possible to find a $G_{2}$ (unless either $|a|=1$ or $|b|=1$ ) such that

$$
\begin{array}{r}
G_{2}\left(U \otimes \mathbf{1}\left(r|00\rangle+s e^{i \phi}|11\rangle\right)=U\left(\begin{array}{cc}
1 & 0 \\
0 & e^{i \phi}
\end{array}\right)|\psi\rangle\left|\chi_{U}\right\rangle\right. \\
G_{2}\left(r|00\rangle+s e^{i \eta}|11\rangle\right)=U\left(\begin{array}{cc}
1 & 0 \\
0 & e^{i \eta}
\end{array}\right)|\psi\rangle|\chi\rangle
\end{array}
$$

Firstly we note again that the state $|\chi\rangle$ cannot depend on $\phi$ or $\eta$ as otherwise the trafo $G_{2}$ would not be linear. However, it may depend on the choice of $U$. Now let us take the scalar product between Eq 58 and Eq. 59. Again $G_{2}$ drops out due to its unitarity and if we use that $g=\left\langle\chi_{U} \mid \chi\right\rangle=1$ to find

$$
|r|^{2} a+|s|^{2} e^{i(\phi-\eta)} a^{*}=\left(a|\alpha|^{2}+a^{*}|\beta|^{2} e^{i(\phi-\eta)}+b \alpha^{*} \beta e^{i \phi}-b^{*} \alpha \beta^{*} e^{-i \eta}\right)
$$

or

$$
\left(|r|^{2}-|\alpha|^{2}\right) a+\left(|s|^{2}-|\beta|^{2}\right) e^{i(\phi-\eta)} a^{*}-b \alpha^{*} \beta e^{i \phi}+b^{*} \alpha \beta^{*} e^{-i \eta}=0
$$

for all $\phi, \eta$. This implies, that

$$
|r|^{2}-|\alpha|^{2}=|s|^{2}-|\beta|^{2}=0 \text { and } b \alpha^{*} \beta=0
$$

Because both $r$ and $s$ are non-zero, we find that also $\alpha$ and $\beta$ are non-zero, which implies that $b=0$ [11. Therefore the only two possible values for $a$ and $b$ are $|a|=1$ and $|b|=1$ and this finishes the proof. 


\section{TRADE-OFF IN RESOURCES}

The results of the previous section allows us to establish the uniqueness of the two teleportable sets which arise in section IV as the two possible cases were the transmission of just one bit from Alice to Bob was sufficient to design a protocol for perfect remote implementation. It should be stressed that the procedure works independently of to which particular subset the transformation belongs to. Imagine now that Alice is given the promise that her apparatus can implement transformations within a particular subset, for instance, any unitary transformation that commutes with the action of the Pauli operator $\sigma_{z}$. In other words, she is provided with a machine that can implement arbitrary rotations around the z-axis. As before, the aim is to implement remotely any such transformation on Bob side, provided that he may hold a qubit state in an arbitrary state $|\psi\rangle_{B}$. We will show in the following that a variation of the protocol discussed in section III allows to implement an arbitrary rotation on Bob side consuming just 1 e-bit and $1 \mathrm{c}$-bit in each direction. In contrast, BQST would consume 1 e-bit and 2 c-bits per state teleportation step. We start with Alice and Bob sharing an e-bit that for concreteness we assume to be the maximally entangled state $|\phi\rangle_{A B}^{+}$. Bob holds a qubit system in an arbitrary state $|\phi\rangle_{B}=\alpha|0\rangle+\beta|1\rangle$. We carry on the same local operations on Bob side described in subsection III.A, that is, a controlled-NOT between Bob's qubits with the unknown state acting as the control qubit followed by a projective measurement of the target qubit in the computational basis. This sequence consumes $1 \mathrm{c}$-bit from Bob to Alice and ends up with both parties sharing the, in general partially entangled, state $\alpha|00\rangle+\beta|11\rangle$. Alice now applies the operation $U_{c}$ onto her qubit followed by a Hadamard transformation. No extra shared entanglement will be required. The global (unnormalized) state of the distributed system after this action can be written as

$$
\begin{aligned}
|\lambda\rangle_{A B} & =\alpha a\left(|0\rangle_{A}+|1\rangle_{A}\right)|0\rangle_{B}+\beta a^{\star}\left(|0\rangle_{A}-|1\rangle_{A}\right)|1\rangle_{B} \\
& =|0\rangle_{A}\left(\alpha a|0\rangle_{B}+\beta a^{\star}|1\rangle_{B}\right)+|1\rangle_{A}\left(\alpha a|0\rangle_{B}-\beta a^{\star}|1\rangle_{B}\right) \\
& =|0\rangle_{A}\left(\alpha U\left(|0\rangle_{B}\right)+\beta U\left(|1\rangle_{B}\right)\right)+|1\rangle_{A}\left(\alpha U\left(|0\rangle_{B}\right)-\beta U\left(|1\rangle_{B}\right)\right)
\end{aligned}
$$

A projective measurement in the computational basis on Alice's side yields a collapsed state on Bob side which is either the correct transformed state by $U_{c}$, whenever the measurement outcome is $|0\rangle_{A}$, or a state that can be locally transformed into the correct one. If the measurement outcome is $|1\rangle_{a}$, all Bob has to do is applying the correcting operation $\sigma_{z}$. Bob needs to know the measurement outcome of Alice's measurement and therefore a further c-bit is consumed in the second part of the protocol. Identical results follow if Alice is given the promise that the transformation $U$ anti-commutes with $\sigma_{z}$. The only difference is that Bob gets the correct transformed state via the application of different correction steps, $\sigma_{x}$ for outcome $|0\rangle_{A}$ and $\sigma_{z} \sigma_{x}$ for outcome $|1\rangle_{A}$.

The explicit construction of a protocol that achieves the remote implementation of any unitary operation of the form $U_{c}$ or $U_{a}$ proves that consuming 1 e-bit and $1 \mathrm{c}$-bit in each direction is sufficient. The necessity can be derived from the following argument. Assume that we can teleport any transformation $U$ which either commutes or anticommutes with $\sigma_{z}$. We can then assume that we could also implement any controlled- $U$ of that form, and in particular we could implement a controlled-NOT operation. But it is known that the non-local implementation of a controlled-NOT requires 1 e-bit and two classical bits in each direction [9], therefore the protocol we have described is optimal.

\section{CONCLUSIONS AND PROSPECTS}

We have analyzed the problem of performing quantum remote control on a qubit. The principles of non-increase of entanglement under LQCC and the impossibility of superluminal communication allow to establish lower bounds on the amount of entanglement and the classical communication cost of a universal remote control protocol: Alice and Bob need to share at least two e-bits and need to communicate no less than two c-bits from Alice to Bob and one c-bit from Bob to Alice. This asymmetry in the communication cost opens the possibility of a different strategy than resorting to bidirectional state teleportation (BQST). While the protocol cannot work perfectly for an arbitrary transformation on a qubit, we have shown here that there are restricted sets of teleportable operations, i.e., operations that can be implemented remotely consuming less overall resources than BQST. Remarkably, up to a local change of basis, only two teleportable subsets exit: Arbitrary rotations around a fixed direction $\vec{n}$ or rotations by a fixed angle around an arbitrary direction lying in a plane orthogonal to $\vec{n}$.

We will finish by describing a possible experimental scenario where the ideas we have developed could be demonstrated. From the practical point view, the most challenging requirement arises from the distribution of a highly entangled state between two remote parties. Nevertheless, theoretical proposals have been made for establishing a maximally entangled state of two trapped ions surrounded by an optical cavity [12]. Let us then assume that a maximally entangled state 
can be created using these techniques. In addition, Bob's cavity holds a second ion initially prepared in a state that for simplicity we will suppose to be an equally weighted superposition of levels $|0\rangle$ and $|1\rangle$. Transformations which either commute or anticommute with the action of $\sigma_{z}$ can be easily realized by means of irradiating Alice's particle with laser light with a suitable value of the ratio $\Delta / \Omega$, where $\Delta$ is the detuning from the atomic transition $|0\rangle \longrightarrow|1\rangle$ and $\Omega$ the laser Rabi frequency. Applying the protocol described in section VII leads to Bob holding a state of the form

$$
|\psi\rangle_{B}=\frac{1}{2}\left(|0\rangle+e^{-i \vartheta}|1\rangle\right)
$$

where $\vartheta$ will be a function of the laser parameters. Therefore, a subsequent measurement of Bob's particle in the $| \pm\rangle$ basis yields a probability for the ion to be found in the $|+\rangle$-state

$$
P_{|+\rangle}=\frac{1+\cos \vartheta}{2}
$$

In other words, under repeated measurements following laser irradiations of different duration on Alice's side, Bob's particle, in a remote location, will exhibit Ramsey fringes. This effect is a nice illustration of how quantum non-locality can be exploited and should lie among the near future experimental capabilities in quantum optics. Applications in quantum communication protocols are foreseeable.

Acknowledgements: We acknowledge discussions with R. Ratonandez and B. Reznik. This work was supported by the UK Engineering and Physical Sciences Research Council (EPSRC), by the EQUIP project of the European Union and the European Science Foundation Programme on 'Quantum Information Theory and Quantum Computing' and by the ESF-QIT conference 'Quantum Information Theory: Theory, Experiment and Perspectives' in Gdansk, 2001.

Note: After this work was completed, we have learned of related results obtained independently by B. Reznik (unpublished) and Chui-Ping Yang and J. Gea-Banacloche, quant-ph/0107100.

\section{APPENDIX}

Here we give the proof that states $|\psi\rangle$ and $\left|\psi_{\perp}\right\rangle$ can be found to satisfy Eqs. (30) and (31) for $\left\langle\psi \mid \psi_{\perp}\right\rangle=0$ and $\left\langle\phi \mid \phi^{\prime}\right\rangle \neq 0$. We drop the superscripts $(n),(m)$ from the operators in these equations and diagonalize the unimodular product $U_{2}^{\dagger} U_{1}$ :

$$
U_{2}^{\dagger} U_{1}\left|\lambda_{ \pm}\right\rangle=e^{ \pm i \lambda}\left|\lambda_{ \pm}\right\rangle
$$

Note that $\lambda \neq 0, \pi, 2 \pi \ldots$ for otherwise $U_{2}^{\dagger} U_{1}= \pm\left(\left|\lambda_{+}\right\rangle\left\langle\lambda_{+}|+| \lambda_{-}\right\rangle\left\langle\lambda_{-}\right|\right)$which is proportional to the identity, and so the operators would be trivially related $U_{2}= \pm U_{1}$ forcing $|\phi\rangle$ and $\left|\phi^{\prime}\right\rangle$ to be orthogonal. Let

$$
\begin{aligned}
|\psi\rangle & =\left(\left|\lambda_{+}\right\rangle+\left|\lambda_{-}\right\rangle\right) / \sqrt{2} \\
\left|\psi_{\perp}\right\rangle & =\left(\left|\lambda_{+}\right\rangle-\left|\lambda_{-}\right\rangle\right) / \sqrt{2}
\end{aligned}
$$

then

$$
\begin{aligned}
U_{2}^{\dagger}|\phi\rangle & =U_{2}^{\dagger} U_{1}|\psi\rangle \\
& =\left(e^{i \lambda}\left|\lambda_{+}\right\rangle+e^{-i \lambda}\left|\lambda_{-}\right\rangle\right) / \sqrt{2}
\end{aligned}
$$

from which we find

$$
|\phi\rangle=U_{2}\left(e^{i \lambda}\left|\lambda_{+}\right\rangle+e^{-i \lambda}\left|\lambda_{-}\right\rangle\right) / \sqrt{2}
$$

As before we also have

$$
U_{2}\left|\psi_{\perp}\right\rangle=\left|\phi^{\prime}\right\rangle
$$

Thus from Eqs. (67), (68) and (66) we get 


$$
\begin{aligned}
\left\langle\phi^{\prime} \mid \phi\right\rangle & =\left\langle\psi _ { \perp } \left| U_{2}^{\dagger} U_{2}\left(e^{i \lambda}\left|\lambda_{+}\right\rangle+e^{-i \lambda}\left|\lambda_{-}\right\rangle\right) / \sqrt{2}\right.\right. \\
& =i \sin (\lambda)
\end{aligned}
$$

which is nonzero (because $\lambda \neq 0, \pi, 2 \pi \ldots$ ).

[1] M.B. Plenio and V. Vedral, Cont. Phys. 39, 431 (1998).

[2] C.H. Bennett, G. Brassard, C. Crepeau, R. Jozsa, A. Peres, and W.K. Wootters, Phys. Rev. Lett. 70, 1895 (1993).

[3] S.F. Huelga, J.A. Vaccaro, A. Chefles and M.B. Plenio, Phys. Rev. A 6304 (4): 2303 (2001); quant-ph/0005061.

[4] W.K. Wootters and W.H. Zurek, Nature 299, 802 (1982).

[5] A. Acín, E. Jané and G. Vidal, quant-ph/0012015.

[6] A.K. Pati, quant-ph/9907022, H.-K. Lo, quant-ph/9912009, C.H. Bennett et al, quant-ph/0006044.

[7] G. Vidal, L. Masanes and J.I.Cirac, quant-ph/0102037

[8] M.A. Nielsen and I.L Chuang, Phys. Rev. Lett. 79, 321 (1997).

[9] J. Eisert, K.A. Jacobs, P. Papadopoulos, and M.B. Plenio, Phys. Rev. A 62, 052317 (2000).

[10] The final correction operation $V$ can be written as $V=W \sigma_{z} W^{-1}$ where $W$ is a generic uni-modular transformation. Eq.(20) can now be re-written as $\sigma_{z} W^{-1} U=e^{i \gamma} W^{-1} U \sigma_{z}$ and the two possible solutions are given by operations of the form $W U_{c}$ or $W U_{a}$. However, this amounts to the trivial degree of freedom of performing a basis change.

[11] Note that it was used explicitly that $G_{1}$ generates an entangled state, ie that $r$ and $s$ are non-zero.

[12] S. van Enk, J.I. Cirac and P. Zoller, Science 279, 205-208 (1998); S. Bose, P.L. Knight, M.B. Plenio and V. Vedral, Phys. Rev. Lett. 83, 5158 - 5161 (1999); L.-M. Duan, M. Lukin, J.I. Cirac, P. Zoller, quant-ph/0105105. 